\title{
Clinical characteristics of COVID-19 infection in a dialysis center during a nosocomial outbreak
}

\author{
Rei lio $^{1}$ - Tetsuya Kaneko ${ }^{1}$ Hitoshi Mizuno ${ }^{1} \cdot$ Yoshitaka Isaka² $^{2}$
}

Received: 31 October 2020 / Accepted: 20 January 2021 / Published online: 8 February 2021

(c) Japanese Society of Nephrology 2021

\begin{abstract}
Background Blood purification therapy is a treatment method, wherein many patients gather in the same space to receive regular treatments, possibly increasing the risk of contracting the coronavirus disease 2019 (COVID-19) through contact, droplet, and aerosol. We experienced a nosocomial outbreak and evaluated the clinical characteristics of COVID-19 infection in patients undergoing blood purification therapy.

Methods We retrospectively analyzed 28 patients who underwent blood purification therapy at the dialysis center of our hospital from April 2, 2020, to April 29, 2020. Logistic regression analysis was performed to identify clinical factors related to COVID-19 for 18 patients who were tested using real-time reverse transcriptase-polymerase chain reaction (RT-PCR).

Results Of the 28 patients, seven were COVID-19 positive, as confirmed by RT-PCR. The median age was 77 years, 22 patients were male (79\%), four patients had acute kidney injury (14\%), and six patients were bedridden (21\%). All infected patients had been admitted to the wards where the nosocomial outbreak had occurred. Logistic regression analysis revealed that being bedridden (odds ratio 13.33, 95\% confidence interval 1.05-169.56, $p<0.05$ ) was significantly related to COVID19 infection. However, the Charlson comorbidity index, receiving dialysis in the same room, and adjacency of the dialysis bed to COVID-19-positive patients before the confirmation of infection did not reveal any significant relationship.

Conclusion Bedridden patients admitted to nosocomial infection wards were associated with COVID-19 infection, and transmission within the dialysis center was not observed. More rigorous infection control measures need to be implemented for bedridden patients undergoing blood purification therapy.
\end{abstract}

Keywords Dialysis $\cdot$ COVID-19 $\cdot$ Nosocomial outbreak $\cdot$ Transmission

\section{Introduction}

An outbreak of the coronavirus disease 2019 (COVID-19) virus started in Wuhan, China, in December 2019 [1]. In Japan, the first domestic case of infection was reported on January 16, 2020, in a patient who had traveled to Wuhan, China. The infection propagated from there, surpassing more than 3,000 domestic cases by April 8,2020. Due to the frequent occurrence of clusters in densely populated areas, a state of emergency was declared on April 16, 2020. By May 8,2020 , the number of cases exceeded 15,000. Meanwhile,

Rei Iio

reiiio.ra@gmail.com

1 Department of Nephrology, Daini Osaka Police Hospital, 2-6-40 Karasugatsuji, Tennoji-ku, Osaka 543-8922, Japan

2 Department of Nephrology, Osaka University Graduate School of Medicine, Suita, Japan the first nosocomial outbreak in Japan was confirmed in February 2020. As hospitals have many elderly patients who are vulnerable to infections, and placed in a densely populated environment, several hospitals have reported nosocomial outbreaks.

The first patient with COVID-19 infection in our hospital was identified on April 12, 2020. The patient was not considered to have COVID-19 infection at the time of admission and did not have advanced kidney disease. Subsequently, a nosocomial outbreak of COVID-19 occurred in two of the seven inpatient wards. Among the patients undergoing blood purification therapy, the first case of COVID-19 was identified on April 17, 2020. COVID-19 is a novel viral infection and, in accordance with the guidelines of the Japanese Association of Dialysis Physicians [2], our dialysis center had been implementing infection control measures. Under the unique circumstances of nosocomial infections, where the risk of secondary infection is extremely high, we used spatial 
and temporal isolation along with the other control measures advocated by the guidelines, as feasible. Patients with a confirmed COVID-19 infection were transferred to another hospital as per the directions from the government for infection control purposes. Finally, there were no hemodialysis (HD) patients at our dialysis center on May 15, 2020. Within a few weeks, a positive COVID-19 infection was confirmed in seven patients who had been undergoing blood purification therapy. Therefore, we considered that it was important to assess factors associated with COVID-19 infection.

Patients undergoing HD are more susceptible to infection than healthy individuals [3]. Additionally, blood purification therapy is a form of treatment wherein many patients gather in a single space to receive treatments for several hours. Therefore, the practice setting of blood purification therapy carries the risk of spreading COVID-19 between patients through medical devices, beds, droplet, or aerosol. Inpatients on dialysis are considered to be at a greater risk of being infected due to their poor general condition. Previous studies have reported viral transmissions in dialysis units. One study reported an influenza virus outbreak in which a patient receiving HD developed an infection which spread throughout the dialysis center [4]. Another study described the spread of the Middle East respiratory syndrome (MERS) to patients with adjacent beds in dialysis units [5]. With regard to COVID-19 infection, a single-center study reported a low likelihood of transmission of COVID-19 infection within dialysis centers [6]. During the outbreaks of influenza virus and MERS, the infection may have spread in the dialysis unit. On the other hand, it has been suggested that current infection control measures may prevent the spread of COVID-19 infection in dialysis units. However, this issue has not yet been fully explored. Therefore, our aim in this study was to evaluate the clinical characteristics associated with COVID-19 infection among patients undergoing blood purification therapy during a nosocomial outbreak in our center.

\section{Methods}

\section{Patients}

This was a retrospective, single-center, cohort study. Seven patients who received blood purification therapy tested COVID-19 positive during a hospital outbreak. The first case among patients undergoing blood purification therapy developed the disease on April 16, 2020, with the diagnosis confirmed by real-time reverse transcription polymerase chain reaction (RT-PCR) on April 17, 2020. The last time infected patient was roomed with another non-infected patients on April 29, 2020. Considering the viral incubation period as 14 days, patients who received treatment at our dialysis center were analyzed from April 2, 2020, to April 29, 2020. For patients admitted before April 2, the baseline was April 2, and for patients admitted after April 2, the baseline was the date of admission. All COVID-19-infected patients who had received blood purification therapy in our center had been discharged or transferred as per government instructions. Patients who had been admitted to the same wards as COVID-19-positive patients and could be discharged were monitored while undergoing outpatient dialysis at our dialysis center after April 16, 2020. These patients were observed until the date of their final outpatient HD session.

\section{RT-PCR}

Public health centers were requested to handle all RT-PCR testing to identify cases of severe acute respiratory syndrome coronavirus 2 (SARS-CoV2). Nasal swab samples were collected from patients. According to the Ministry of Health, Labor, and Welfare's "Consultation Criteria for COVID-19 Infections", issued on February 17, 2020, a suspected case of COVID-19 is defined as a fever $\geq 37.5^{\circ} \mathrm{C}$ and respiratory symptoms lasting for 2 days in patients with underlying diseases. During the observation period, RT-PCR test was performed when these symptoms developed, especially in suspected cases, as nosocomial infections were occurring in our hospital. After April 22, 2020, all patients underwent RT-PCR testing sequentially due to the nosocomial outbreak of the COVID-19 infection. RT-PCR testing was also completed for all hospital staff.

\section{Data collection}

The date of incidence of COVID-19 was defined as the date of symptom onset for symptomatic patients and the date of RT-PCR testing for asymptomatic patients. To investigate the involvement of aerosol and droplet infections in the dialysis center, we assessed the number of dialysis sessions in the same room and the adjacency of dialysis beds within 2,7 , and 14 days of the dates of COVID-19 symptom onset. The number of dialysis sessions was defined as the count until the dates of symptom onset among COVID-19-positive patients or until the last dialysis day among COVID-19-negative patients. Patients' level of awareness and degree of independence were assessed by hospital nurses using the national assessment tool for determining long-term insurance to ascertain the impact of contact or droplet infection [7]. The age at baseline, sex, body mass index, smoking history, dialysis vintage, vascular access, admission to the nosocomial infection ward, use of angiotensin-converting enzyme inhibitors or angiotensin II receptor blocker, the purpose for hospital admission, history of hypertension, and the presence of diabetes and neoplastic complications were extracted from the patients' medical records. Through 
hematological examinations, we obtained the white blood cell count, lymphocyte count, serum lactate dehydrogenase (LDH) level, serum albumin level, and serum C-reactive protein (CRP) level at baseline and at the time of positive RT-PCR confirmation of COVID-19 infection. Symptoms that began 1 week before the diagnosis, including the date of confirmation of a positive COVID-19 test, were extracted as early onset symptoms, including fever, cough, shortness of breath, mild fever, malaise, diarrhea, and appetite loss.

\section{Infection control}

Our dialysis center had 14 HD beds. As the infection spread increasingly through the community, our hospital adopted infection control measures. Even before the COVID-19 outbreak, our center had been practicing daily infection control measures according to the guideline published by the Japanese Association of Dialysis Physicians in 2015 [8]. Following publication of "The Japanese Association of Dialysis Physicians. The 4th issue on the measures against COVID19 in dialysis facilities [in Japanese]" on April 3, 2020 [2], we implemented required infection control measures, as summarized in Table 1.

\section{Statistical analysis}

Continuous variables are shown as median (interquartile range) and nominal variables as numerical values (\%). Univariate logistic regression analysis was performed to identify clinical factors related to COVID-19 among 18 patients who were tested using RT-PCR. Stata IC version 15.1 (StataCorp,

Table 1 Infection control measures for COVID-19 in our dialysis center

Infection control in our dialysis center

Overall measures

1. Standard infection control measures were appropriately performed by all HCWs in the dialysis center

2. All patients were asked to wear face masks

Sanitation and environmental measures

3. The HD beds used were disinfected with hypochlorous acid solution after each patient's treatment

4. We provided additional ventilation by opening windows to the extent possible. The mechanical ventilation rate of the center was six times/h, and the vents were located on the ceiling above patients' bed. There was also a supply vent above patients' head and an exhaust vent above patients' feet. The air flowed in one direction and did not cross over several patients

5. We increased the space between patient beds when there were few patients in the dialysis center. In our center, the space between dialysis beds was $0.8-1.2 \mathrm{~m}$

For patients suspected of COVID-19 infection

6. According to the Ministry of Health, Labor, and Welfare's "Consultation Criteria for COVID-19 Infections" issued on February 17, 2020, a suspected case of COVID-19 is defined as a fever $\geq 37.5^{\circ} \mathrm{C}$ and respiratory symptoms lasting for two days in patients with underlying diseases. During the period of observation, an RT-PCR test was performed when these symptoms appeared

7. Patients presenting with fever and respiratory symptoms were tested using RT-PCR. If positive, isolated HD was performed, and if negative, HD was performed with adequate infection control measures in place, including sufficient spacing between beds

8. Anytime a patient suspected of COVID-19 infection or his/her caregiver entered or exited the dialysis center, surfaces touched and used by these individuals were cleaned with a hypochlorous acid solution

For COVID-19 patients

9. Patients with positive RT-PCR results were advised to transfer to another hospital dedicated to coronavirus treatment immediately and continued to receive individual and isolated HD while awaiting the transfer

For patients who underwent outpatient dialysis

10. Patients who were admitted to the same wards as COVID-19 positive patients and could be discharged were monitored for 14 days of health status while receiving outpatient dialysis at our hospital after April 16, 2020

11. Outpatients were asked to record their body temperature and symptoms at home every day and to call the hospital before a visit if they noticed COVID-19 symptoms or a fever above $37.5^{\circ} \mathrm{C}$

12. Patients with symptoms were examined before entering the dialysis center

13. Sufficient spacing between beds was ensured

14. We asked outpatients to come to the center in clothes that were easy to remove. Patients changed on the bed, behind a curtain. Change rooms in the dialysis unit were closed

15. Body weight was measured for each patient at each bed, with no sharing of weight scales between patients

16. To further minimize contact between patients, the usual emergency exit was used as an entrance for outpatients

17. Patient transportation was performed by the family

COVID-19 coronavirus disease 2019, HCWs health care workers, $H D$ hemodialysis, $R T-P C R$ reverse transcription polymerase chain reaction 
College Station, Texas) was used for all statistical analyses, with $p<0.05$ indicating a statistically significant.

\section{Results}

Between April 2, 2020, and April 29, 2020, 25 inpatients and 29 health care workers (HCWs) in our hospital were infected with COVID-19, the spread of infection occurring principally in two of our seven hospital wards. During the observation period, 28 patients received treatment at our dialysis center, with COVID-19 infection confirmed in seven of these patients, and RT-PCR test rate was 64\% (18/28). Of these seven patients, six were symptomatic and only one was asymptomatic. The median age of the 28 patients at baseline was 77 years, and 22 patients (79\%) were male. Fifteen patients (54\%) had diabetes as a comorbidity, four (14\%) had acute kidney injury (AKI), six (21\%) had severe dementia, one (4\%) underwent a peripheral blood stem cell collection, and six (21\%) patients were bedridden. All infected patients undergoing blood purification therapy had been admitted to two wards where the nosocomial outbreak occurred. None of the COVID-19-negative patients receiving blood purification therapy were found to be infected during the first 2 weeks after discharge. None of the patients with a first negative RT-PCR result tested positively on re-examination. The characteristics of these patients are summarized in Table 2.

We examined the factors related to COVID-19 infection of the patients undergoing blood purification therapy using univariate logistic regression analysis (Table 3). Highly dependent (bedridden) patients were associated with COVID-19 infection (odds ratio [OR] 13.33, 95\% confidence interval (95\% CI) 1.05-169.56, $p<0.05$ ). However, the Charlson comorbidity index was unrelated to COVID-19 infection.

Table 2 Clinical characteristics of patients who underwent blood purification therapy

\begin{tabular}{|c|c|c|c|c|}
\hline Characteristics & All $n=28$ & $\begin{array}{l}\text { SARS-CoV-2 positive } \\
n=7\end{array}$ & $\begin{array}{l}\text { SARS-CoV-2 negative } \\
n=11\end{array}$ & $\begin{array}{l}\text { RT-PCR not } \\
\text { performed } \\
n=10\end{array}$ \\
\hline Age (years) & $77[63-81]$ & $81[59-83]$ & 78 [70-79] & $67[60-79]$ \\
\hline Sex, male $n(\%)$ & $22(79)$ & $5(71)$ & $9(82)$ & $8(80)$ \\
\hline Body mass index $\left(\mathrm{kg} / \mathrm{m}^{2}\right)$ & $21[19-25]$ & 20 [19-22] & $20[19-21]$ & $24[22-28]$ \\
\hline \multicolumn{5}{|l|}{ Purpose of admission $n(\%)$} \\
\hline Initiation of HD & $9(32)$ & $3(43)$ & $2(18)$ & $4(40)$ \\
\hline Treatment for complicated disease & $14(50)$ & $1(14)$ & $8(73)$ & $5(50)$ \\
\hline Therapy for AKI & $4(14)$ & $3(43)$ & $1(9)$ & $0(0)$ \\
\hline Others & $1(4)$ & $0(0)$ & $0(0)$ & $1(10)$ \\
\hline Dialysis vintage (months) & $7[0-53]$ & $0[0-1]$ & 17 [1-97] & $11[0-60]$ \\
\hline Dialysis sessions (counts) & $7[3-9]$ & 7 [3-9] & $9[8-12]$ & $3[2-4]$ \\
\hline $\mathrm{AKI} n(\%)$ & $4(14)$ & $3(43)$ & $1(9)$ & $0(0)$ \\
\hline Hypertension $n(\%)$ & $24(86)$ & $7(100)$ & $10(91)$ & $7(70)$ \\
\hline Diabetes mellitus $n(\%)$ & $15(54)$ & $6(86)$ & $4(36)$ & $5(50)$ \\
\hline Coronary artery disease $n(\%)$ & $8(29)$ & $2(29)$ & $5(45)$ & $1(10)$ \\
\hline Malignancy current $n(\%)$ & $6(21)$ & $1(14)$ & $2(18)$ & $3(30)$ \\
\hline Smoking history $n(\%)$ & $24(86)$ & $7(100)$ & $10(91)$ & $7(70)$ \\
\hline ACEi or ARB use $n(\%)$ & $8(29)$ & $2(29)$ & $3(27)$ & $3(30)$ \\
\hline \multicolumn{5}{|l|}{ Dialysis access $n(\%)$} \\
\hline Dialysis catheter use & $8(29)$ & $4(57)$ & $3(27)$ & $1(10)$ \\
\hline \multicolumn{5}{|l|}{ ADL status } \\
\hline Charlson comorbidity index & $6[2-8]$ & $6[5-8]$ & $5[2-9]$ & $5[2-7]$ \\
\hline Charlson comorbidity index $\geq 5, n(\%)$ & $22(79)$ & $7(100)$ & $6(55)$ & $9(90)$ \\
\hline Dementia severe $n(\%)$ & $6(21)$ & $3(43)$ & $2(18)$ & $1(10)$ \\
\hline Level of dependency (medium and high) $n(\%)$ & $12(43)$ & $6(86)$ & $4(36)$ & $2(20)$ \\
\hline Level of dependency (high) $n(\%)$ & $6(21)$ & $4(5)$ & $1(9)$ & $1(10)$ \\
\hline Ward with nosocomial infection $n(\%)$ & $16(57)$ & $7(100)$ & $5(45)$ & $4(40)$ \\
\hline Admission to a private room $n(\%)$ & $5(18)$ & $3(43)$ & $2(18)$ & $0(0)$ \\
\hline
\end{tabular}

Data are presented as $n(\%)$ or median [interquartile range]

$S A R S-C o V-2$ severe acute respiratory syndrome coronavirus $2, R T-P C R$ reverse transcription polymerase chain reaction, $H D$ hemodialysis, $A K I$ acute kidney injury, $A C E i$ angiotensin-converting enzyme inhibitors, $A R B$ angiotensin II receptor blocker, $A D L$ activity of daily living 
Table 3 Logistic regression analysis for COVID-19 infection

\begin{tabular}{|c|c|c|}
\hline Variables & Odds ratio $(95 \% \mathrm{CI})$ & $p$ value \\
\hline \multicolumn{3}{|l|}{ Coexisting conditions } \\
\hline Diabetes mellitus & $10.50(0.91-121.39)$ & 0.06 \\
\hline AKI & $7.50(0.60-95.38)$ & 0.12 \\
\hline Dialysis catheter use & $3.56(0.48-26.28)$ & 0.21 \\
\hline \multicolumn{3}{|l|}{ ADL-related status } \\
\hline Charlson comorbidity index & $1.34(0.81-2.24)$ & 0.26 \\
\hline Severe dementia & $3.38(0.40-28.74)$ & 0.27 \\
\hline $\begin{array}{l}\text { Level of dependency (medium and } \\
\text { high) }\end{array}$ & $10.50(0.91-121.39)$ & 0.06 \\
\hline Level of dependency (high) & $13.33(1.05-169.56)$ & $<0.05$ \\
\hline Admission to a private room & $3.38(0.40-28.74)$ & 0.27 \\
\hline
\end{tabular}

COVID-19 coronavirus disease 2019, CI confidence interval, AKI acute kidney injury, $A D L$ activity of daily living

Whether the patient was admitted to a private room or a large room with several other patients in the hospital ward was unrelated to the risk of infection. Therefore, for patients undergoing blood purification therapy, being bedridden (and thus receiving more care from $\mathrm{HCWs}$ ) increases the risk of COVID-19 infection, underlining the importance of contact or droplet infection control measures. All infected patients had been admitted to the wards where the nosocomial infections occurred, suggesting the possibility of contact or droplet infection on the wards. To specifically assess the transmission of COVID-19 infection in the dialysis center, we analyzed the adjacency of beds with COVID-19-infected patients in the dialysis center and the number of HD sessions carried out in the same room before COVID-19 confirmation (Table 4). Bed adjacency with infected patients within 14 days before symptom onset was not related to COVID-19 prevalence (OR 0.39, 95\% CI 0.12-1.27, $p=0.12$ ). COVID19 infection was also not associated with the number of HD sessions (OR $0.75,95 \%$ CI $0.54-1.04, p=0.08$ ). We also examined whether bed adjacency and HD sessions in the same room with infected patients within 2 or 7 days before symptom onset were associated with COVID-19 infection; however, none of these were associated with susceptibility to COVID-19 infection. Thus, there is little likelihood of COVID-19 infection transmission through medical device, beds, droplet, or aerosol in the dialysis center.

As this was a nosocomial outbreak, cases infected with COVID-19 were able to identify symptoms before the onset of the disease, and blood samples could be collected early in the development of the disease. Therefore, to identify the early characteristics of nosocomial infections, we compared the early symptoms of COVID-19 and laboratory values between the seven patients with RT-PCR-verified COVID-19 infection and the 11 patients with negative RT-PCR results (Table 5). While fever (5 of 7 vs. 0 of 1 , for the COVID19-positive and COVID-19-negative group, respectively, $p<0.01$ ) and cough ( 5 of 7 vs. 1 of 11 , respectively, $p<0.01$ ) were identified as early symptoms, shortness of breath was not associated with COVID-19. The absence of an association between shortness of breath and COVID-19 infection likely reflects the low physical activity status of these patients (who were bedridden). Appetite loss was associated with COVID-19 infection (4 of 7 vs. 1 of 11, respectively, $p<0.05$ ). Malaise (7 of 7 vs. 7 of $11, p=0.12$ ) and mild fever ( 6 of 7 vs. 5 of $11, p=0.15$ ) tended to be associated with COVID-19. The results of blood tests conducted during the early stages of the infection showed elevated serum CRP levels ( 1.99 vs. $0.54 \mathrm{mg} / \mathrm{dL}$, respectively, $p<0.01$ ), and high serum LDH (227 vs. $175 \mathrm{mg} / \mathrm{dL}$, respectively, $p=0.09$ ) levels (Table 5).

Figure 1 shows the date(s) of HD sessions, symptom onset, COVID-19 confirmation, and the final group HD session in the dialysis center for the seven patients with a confirmed COVID-19 infection. The clinical characteristics of these seven patients are summarized in Supplementary Table 1. Patients A, B, and C had AKI induced by hemorrhagic shock (A), during acute heart failure (B), and after cardiovascular surgery (C). These patients no longer received group $\mathrm{HD}$ at the time of COVID-19 positive confirmation. Patients D, E, F, and G had chronic kidney
Table 4 Logistic regression analysis for COVID-19 infection in the situation of the dialysis center

\begin{tabular}{lll}
\hline Variable & Odds ratio (95\% CI) & $p$ value \\
\hline HD sessions (count) & $0.75(0.54-1.04)$ & 0.08 \\
Bed adjacency with a future COVID-19-positive patient (count) & & \\
Bed adjacency for 14 days prior to the diagnosis & $0.39(0.12-1.27)$ & 0.12 \\
Bed adjacency for 7 days prior to the diagnosis & $0.28(0.06-1.33)$ & 0.11 \\
Bed adjacency for 2 days prior to the diagnosis & $0.31(0.03-2.80)$ & 0.30 \\
Same room with a future COVID-19-positive patient (count) & & \\
Same room for 14 days prior to the diagnosis & $0.78(0.58-1.06)$ & 0.12 \\
Same room for 7 days prior to the diagnosis & $0.67(0.43-1.02)$ & 0.06 \\
Same room for 2 days prior to the diagnosis & $0.56(0.31-1.01)$ & 0.05 \\
\hline
\end{tabular}

COVID-19 coronavirus disease 2019, CI confidence interval, $H D$ hemodialysis 
Table 5 Early clinical manifestations when RT-PCR positive for COVID-19 was confirmed

\begin{tabular}{|c|c|c|c|c|}
\hline Characteristics & All $n=18$ & SARS-CoV-2 positive $n=7$ & SARS-CoV-2 negative $n=11$ & $p$ value \\
\hline \multicolumn{5}{|l|}{ Preceding signs or symptoms } \\
\hline \multicolumn{5}{|l|}{ Typical symptoms } \\
\hline Fever, $n(\%)$ & $5(28)$ & $5(71)$ & $0(0)$ & $<0.01$ \\
\hline Cough, $n(\%)$ & $6(33)$ & $5(71)$ & $1(9)$ & $<0.01$ \\
\hline Shortness of breath, $n(\%)$ & $3(17)$ & $2(29)$ & $1(9)$ & 0.53 \\
\hline \multicolumn{5}{|l|}{ Atypical symptoms } \\
\hline Subfever, $n(\%)$ & $11(61)$ & $6(86)$ & $5(45)$ & 0.15 \\
\hline Malaise, $n(\%)$ & $14(78)$ & $7(100)$ & $7(64)$ & 0.12 \\
\hline Diarrhea, $n(\%)$ & $6(33)$ & $3(43)$ & $3(27)$ & 0.63 \\
\hline Appetite loss, $n(\%)$ & $5(28)$ & $4(57)$ & $1(9)$ & $<0.05$ \\
\hline \multicolumn{5}{|c|}{ Laboratory data at the time of COVID-19 detection } \\
\hline White blood cell count $(/ \mu \mathrm{L})$ & $5500[2300-15500]$ & $5500[2300-15500]$ & $5850[2700-8600]$ & 0.49 \\
\hline Lymphocyte count $(/ \mu \mathrm{L})$ & 799 [417-4728] & $765[439-4728]$ & $942[417-1901]$ & 0.63 \\
\hline Serum albumin $(\mathrm{g} / \mathrm{dL})$ & $2.6[1.6-3.7]$ & $2.4[1.6-2.8]$ & $2.7[1.7-3.7]$ & 0.15 \\
\hline Serum LDH (mg/dL) & 214 [106-480] & $227[127-480]$ & $175[106-220]$ & 0.09 \\
\hline Serum CRP (mg/dL) & $1.07[0.1-7.97]$ & $1.99[0.73-7.97]$ & $0.54[0.1-6.72]$ & $<0.01$ \\
\hline
\end{tabular}

Data are $n(\%)$ and median [interquartile range]

COVID-19 coronavirus disease 2019, SARS-CoV-2 severe acute respiratory syndrome coronavirus 2, $A D L$ activity of daily living, $C R P$ C-reactive protein
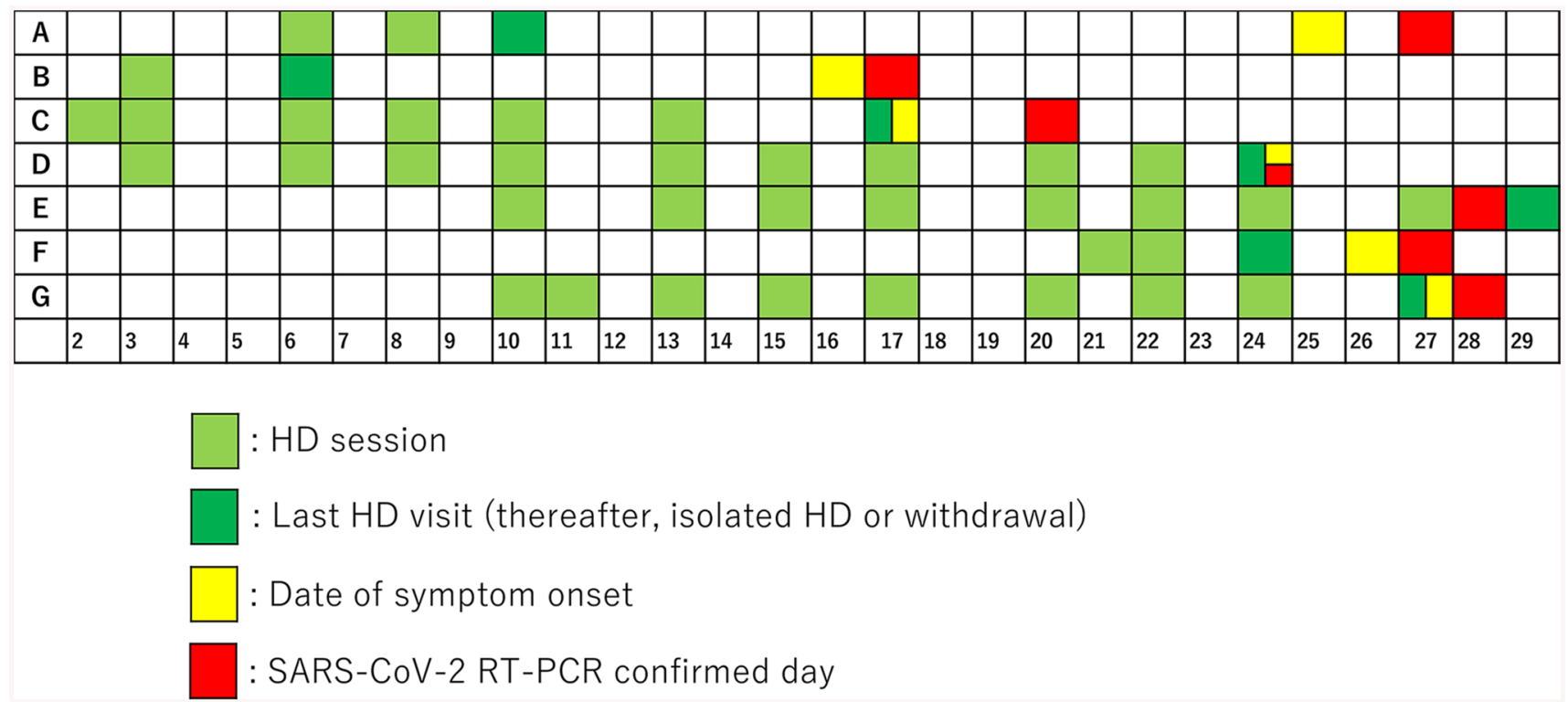

Fig. 1 Summary of days until confirmation of a positive real-time reverse transcriptase-polymerase chain reaction (RT-PCR) for COVID-19 for patients treated in the dialysis center after April 2 , 2020. The color coding is as follows: light green, date that the

disease and were receiving maintenance HD. Patients F and $\mathrm{G}$ had been in the introduction period for HD. Patients D, E, F, and G were infected within a few days. Patients D and $\mathrm{G}$ were wheelchair-bound, $\mathrm{E}$ was bedridden, and $\mathrm{F}$ had a severe visual impairment. These four patients, therefore, patients visited our center for blood purification therapy; dark green, last day of a patient's treatment in our dialysis center; yellow, date of symptom onset; and red, date of positive RT-PCR confirmation

required extensive personal assistance, but had no contact with each other.

The staff of the dialysis center included three physicians, seven nurses, and three medical engineers. These 
individuals were not infected with COVID-19 during the observation period.

\section{Discussion}

In a dialysis center, once a single HD patient becomes infected with COVID-19, many patients who receive treatments during the same timeframe will be at risk of droplet, aerosol, or contact infection through medical devices, beds, patients, and HCWs. Although previous studies have reported on the risk of COVID-19 infection among dialysis patients [6,9-12], only a few have discussed the route of infection and, thus, it is unclear what measures should be focused on. We experienced a nosocomial infection that spread within a few weeks and investigated the characteristics of COVID-19 infection in patients undergoing blood purification therapy. Our findings indicate that COVID-19 infection is unlikely to be transmitted with a dialysis center, but were transmitted to patients with reduced activities of daily living (ADL), suggesting the spread of infections in nosocomial wards or during transportation. Furthermore, amid a nosocomial outbreak, any symptoms in these patients, such as fever, cough, loss of appetite, and elevation of serum CRP level, were likely signs of COVID- 19.

We found that the COVID-19 infection was significantly more prevalent among patients who required more care and medical attention from HCWs, with no association between the Charlson comorbidity index and the risk of infection. The need for care increases the chance of infection by contact or droplet. The risk of contact infection is also high in bedridden patients who require more support from HCWs, such as being transported to the dialysis center on a stretcher or wheelchair. As SARS-CoV2 tends to remain in the environment [13], infection control measures, such as disinfection of environmental surfaces, in addition to hand sanitation procedures advocated by the World Health Organization [14], must be carried out in an extremely stringent manner.

In our analysis, COVID-19 infection was not found to be associated with the number of HD sessions. Furthermore, dialysis in the same room and the adjacency of beds with patients infected with COVID-19 was not associated with infection, suggesting that COVID-19 is unlikely to be transmitted through droplets or aerosols between patients in the dialysis center, which is consistent with a previous report [6]. Several factors can explain this. First, in a dialysis center dedicated to inpatients, there is no need for change rooms or resting areas where prolonged contact between patients could occur. Therefore, inpatient dialysis units are fundamentally different from home and other public venues [15]. Second, all patients wore masks which decreased the likelihood of aerosol and droplet transmission between patients [16]. Third, while reports indicate that ventilation is vital for minimizing aerosol infections [17], the Japanese Association of Dialysis Physicians advocates for dialysis centers automatic ventilating six times per hour. In our center, air flowed in one direction and, thus, did not cross between patients (see Table 1 for an explanation). Fourth, the fact that no HCWs in the dialysis center were infected with COVID19 is another reason the infection did not spread within the dialysis center. Hence, we believe that by wearing masks, implementing automatic ventilation, providing additional ventilation by opening the windows, and preventing infection in HCWs, we considerably reduced the risk of droplet or aerosol transmission of COVID-19. Furthermore, to prevent the spread of nosocomial infection, it is crucial to detect and isolate the infected patients as soon as possible.

The early symptoms of COVID-19 infection among our patients were consistent with widely known symptoms of fever and cough. However, many patients did not develop breathing difficulties. It has previously been reported that some patients on HD tend not to show symptoms of COVID19 infection [11] and, thus, should be carefully monitored. CRP was the only laboratory parameter that was significantly associated with COVID-19 infection. The median CRP level of infected patients at the time of disease onset was $1.99 \mathrm{mg} / \mathrm{dL}$. Close attention should be paid to serum CRP level changes, regardless of whether they are high or low.

This study had some limitations. First, this was a singlecenter cohort analysis and, thus, lacked statistical power due to the small number of cases included. Second, not all patients received an RT-PCR test. Third, because SARSCoV2 is known to be a virus that mutates quickly, it is unclear whether the same infection control measures will suffice going forward.

\section{Conclusion}

This study showed that patients on blood purification therapy who were bedridden and admitted to wards occurring a nosocomial outbreak were more likely to be infected with COVID-19. There was no evidence of transmission of infection within the dialysis center. Strict infection control measures should be implemented, especially for bedridden patients.

Supplementary Information The online version contains supplementary material available at https://doi.org/10.1007/s10157-021-02025-8.

Acknowledgements We would like to thank dialysis center staff for working so diligently amid the difficulties of nosocomial transmission of COVID-19. We also would like to thank the hospitals and clinics that accepted our patients when they were transferred or discharged from our hospital. 
Author contributions RI managed the patients, made decisions regarding the patients' treatment, and wrote the whole manuscript. KT is a director at our department and checked the manuscript. MH is a nephrologist at our hospital and managed patients. YI provided scientific advice and revised the manuscript. All authors have read and approved the manuscript.

\section{Compliance with ethical standards}

Conflict of interest The authors declare that they have no competing interests.

Ethical approval This study was approved by the Ethics Committee at Daini Osaka Police Hospital (1025) and was conducted according to the ethical principles of the Declaration of Helsinki.

Informed consent Informed consent was obtained from all individual participants included in the study.

\section{References}

1. Zhu N, Zhang D, Wang W, Li X, Yang B, Song J, et al. A novel Coronavirus from patients with pneumonia in China, 2019. N Engl J Med. 2020;382:727-33.

2. COVID-19 Task Force Committee of the Japanese Association of Dialysis Physicians; Japanese Society for Dialysis Therapy; Japanese Society of Nephrology, Kikuchi K, Nangaku M, Ryuzaki M, Yamakawa T, Hanafusa N, Sakai K, Kanno Y, et al. COVID19 of dialysis patients in Japan: current status and guidance on preventive measures. Ther Apher Dial. 2020;24:361-5.

3. Wakasugi M, Kawamura K, Yamamoto S, Kazama JJ, Narita I. High mortality rate of infectious diseases in dialysis patients: a comparison with the general population in Japan. Ther Apher Dial. 2012;16:226-31.

4. Hotchkiss JR, Holley P, Crooke PS. Analyzing pathogen transmission in the dialysis unit: time for a (schedule) change? Clin J Am Soc Nephrol. 2007;2:1176-85.

5. Assiri A, McGeer A, Perl TM, Price CS, Al Rabeeah AA, Cummings DA, et al. Hospital outbreak of Middle East respiratory syndrome coronavirus. N Engl J Med. 2013;369:407-16.

6. Rincon A, Moresco F, Lopez-Herradon A, Fernandez-Robres MA, Cidraque I, Nin J, et al. The keys to control a COVID-19 outbreak in a hemodialysis unit. Clin Kidney J. 2020;24:361-5.
7. Tsutsui T, Muramatsu N. Care-needs certification in the long-term care insurance system of Japan. J Am Geriatr Soc. 2005;53:522-7.

8. Guidelines for Standard Hemodialysis Procedure and Prevention of Infection in Maintenance Hemodialysis Facilities (4th edition). The Japanese Society of for Dialysis Therapy. 2015. http://www. touseki-ikai.or.jp/htm/07_manual/doc/20150512_infection_guide line_ver4.pdf. Accessed 22 Sept 2020.

9. Clarke C, Prendecki M, Dhutia A, Ali MA, Sajjad H, Shivakumar O, et al. High prevalence of asymptomatic COVID-19 infection in hemodialysis patients detected using serologic screening. J Am Soc Nephrol. 2020;31:1969-75.

10. Cho JH, Kang SH, Park HC, Kim DK, Lee SH, Do JY, et al. Hemodialysis with cohort isolation to prevent secondary transmission during a COVID-19 outbreak in Korea. J Am Soc Nephrol. 2020;31:1398-408.

11. Tang H, Tian JB, Dong JW, Tang XT, Yan ZY, Zhao YY, et al. Serologic detection of SARS-CoV-2 infections in hemodialysis centers: a multicenter retrospective study in Wuhan, China. Am J Kidney Dis. 2020;76:490-9.

12. Valeri AM, Robbins-Juarez SY, Stevens JS, Ahn W, Rao MK, Radhakrishnan J, et al. Presentation and outcomes of patients with ESKD and COVID-19. J Am Soc Nephrol. 2020;31:1409-15.

13. van Doremalen N, Bushmaker T, Morris DH, Holbrook MG, Gamble A, Williamson BN, et al. Aerosol and surface stability of SARS-CoV-2 as compared with SARS-CoV-1. N Engl J Med. 2020;382:1564-7.

14. World Health Organization. WHO guidelines on hand hygiene in health care. 2009. https://apps.who.int/iris/bitstream/handle/10665 /44102/9789241597906_eng.pdf?sequence=1. Accessed 22 Sept 2020.

15. Li W, Zhang B, Lu J, Liu S, Chang Z, Cao P, et al. The characteristics of household transmission of COVID-19. Clin Infect Dis. 2020. https://doi.org/10.1093/cid/ciaa450.

16. Chu DK, Akl EA, Duda S, Solo K, Yaacoub S, Schünemann HJ, et al. Physical distancing, face masks, and eye protection to prevent person-to-person transmission of SARS-CoV-2 and COVID-19: a systematic review and meta-analysis. Lancet. 2020;395:1973-87.

17. Morawska L, Tang JW, Bahnfleth W, Bluyssen PM, Boerstra A, Buonanno $\mathrm{G}$, et al. How can airborne transmission of COVID-19 indoors be minimised? Environ Int. 2020;142:105832.

Publisher's Note Springer Nature remains neutral with regard to jurisdictional claims in published maps and institutional affiliations. 